\title{
Aging without disorder on long time scales
}

\author{
Werner Krauth $^{(1)}$ and Marc Mézard ${ }^{(2)}$ \\ (1) CNRS-Laboratoire de Physique Statistique de l'ENS \\ 24, rue Lhomond; F-75231 Paris Cedex 05; France \\ e-mail: krauth@physique.ens.fr \\ (2) CNRS-Laboratoire de Physique Théorique de l'ENS* \\ 24, rue Lhomond; F-75231 Paris Cedex 05; France \\ e-mail: mezard@physique.ens.fr
}

(July 1994)

We study the Metropolis dynamics of a simple spin system without disorder, which exhibits glassy dynamics at low temperatures. We use an implementation of the algorithm of Bortz, Kalos and Lebowitz [1]. This method turns out to be very efficient for the study of glassy systems, which get trapped in local minima on many different time scales. We find strong evidence of aging effects at low temperatures. We relate these effects to the distribution function of the trapping times of single configurations.

LPTENS preprint 94/20.

PACS numbers: 02.70Lq, 64.70Pf, 64.650Cn

* Unité propre du CNRS, associée à l'ENS et à l'Université de Paris XI 
In the last few years, a lot of work has been devoted to the description of systems with glassy dynamics. The phenomenon of aging [2] seems to be ubiquitous. Dynamical aging, which may be loosely defined as the property that correlation or response functions on a time scale $\tau$ depend on the age of the system $t_{w}$ when $\tau \simeq t_{w}$, has been used as a powerful probe in experimental studies of the spin glass phase [3]. Recently, a lot of numerical studies of various spin glass systems have reported aging effects [4]. The theoretical understanding of aging is still not satisfactory, although there have been interesting proposals of phenomenological models [6 8], and also some attempts at a microscopic study and a link with the static replica solution of some simple mean field systems [9] [13].

In the spin glass case, slow dynamics is generated by the frozen disorder. In this paper we want to report on the numerical observation of aging effects in a model which has no quenched disorder. This model is a simple spin system with long-range four-spin interactions which has been studied from a physical point of view by Bernasconi [5]. That quenched disorder is not mandatory for the existence of a glassy dynamics is evident: the glassy state is a notorious counter example. The system we study provides a simple model for this effect. In a sense, the system generates its own disorder through the slowing down of some degrees of freedom. This model can help to provide a new bridge between glassy systems with or without quenched disorder, on top of the approaches through mode coupling theory [14, or some other glass models studied recently [15]. The emergence of long time scales in frustrated systems without disorder has been noticed in recent papers [16].

The numerical observation of some non-equilibrium phenomena which resemble aging in a Monte Carlo simulation is rather easy. A more difficult task is to assert whether these are just transient phenomena taking place on time scales shorter than some equilibration time $t_{e q}$ or if $t_{e q}$ is infinite. This task is difficult almost by definition: in the glass phase the dynamics is slow, and aging should be demonstrated by the existence of relaxation processes taking place on very different time scales, in order to be safely distinguished from a simple, but slow relaxation rate towards equilibrium. This requires simulating a system at low temperatures, in a regime where the acceptance rate of the Metropolis algorithm becomes very small.

This problem of the low acceptance probability can be circumvented efficiently with an algorithm, originally proposed by Bortz et al [1], and used in some rare occasions [16] [17]. We introduce here an efficient implementation of this algorithm, and apply it to the Bernasconi model. At low temperatures, we can simulate this system on time scales which are several orders of magnitude larger than those accessible by a straight Monte Carlo simulation. We suspect that this algorithm could be quite useful for the simulations of a whole class of glassy systems at low temperatures, which we shall characterize briefly in the conclusion.

The problem studied by Bernasconi some years ago consists in finding low autocorrelation binary sequences (LABS). This optimization problem, originally introduced for its applications in communications, can be turned into a physical problem of $\mathrm{N}$ Ising spins, where the $2^{N}$ spin configurations are weighted by a Boltzmann factor, with an energy given by:

$$
\mathcal{H}=\frac{1}{N} \sum_{k=1}^{N-1}\left[\sum_{i=1}^{N-k} S_{i} S_{i+k}\right]^{2}
$$

Monte Carlo simulations have been carried out by Bernasconi [5], Golay [18], and more 
recently by Migliorini [19], who have shown that finding the ground state (or low lying configurations) is numerically hard; the system freezes at a certain temperature, below which the specific heat becomes very small: the system gets trapped into metastable states. Here we would like to consider it as a simple prototype of systems exhibiting dynamical aging and undergoing a glass transition. Simultaneously to our numerical simulations, several independent works have tried to investigate this problem [20], or another version with periodic boundary conditions [21], analytically by approximating it by a disordered system. The corresponding disordered problem can be studied by the replica technique and undergoes a "one step replica symmetry breaking" phase transition characteristic of a class of spin glass transitions of the category of the Random Energy Model [22,223]. Such a freezing scenario is well confirmed by our simulations: at low temperatures, the system gets trapped into single configurations which it takes many Monte Carlo steps to escape from.

Let us now describe the algorithm we have been using. We adopt the usual single spin-flip dynamics, which produces a sequence of configurations

$$
\sigma_{1}\left(\tau_{1}\right) \rightarrow \sigma_{2}\left(\tau_{2}\right) \ldots \rightarrow \sigma_{i}\left(\tau_{i}\right) \rightarrow \sigma_{i+1}\left(\tau_{i+1}\right) \ldots \text { etc }
$$

In eq. (2), the symbols indicate that the system stays in configuration $\sigma_{i}$ for a time $\tau_{i}$ etc. The transition probability is non-zero only for configurations differing by a single spin. (We denote in the following by $\sigma^{[m]}$ the configuration which results from $\sigma$ after flipping spin $m$ ). We use the Metropolis dynamics, in which the transition probability per unit time $\Delta \tau$ is given by the explicit formula:

$$
p\left(\sigma \rightarrow \sigma^{[m]}\right)=\frac{\Delta \tau}{N}\left\{\begin{array}{l}
1 \quad\left(i f \quad E\left(\sigma^{[m]}\right)<E(\sigma)\right) \\
\exp \left[-\beta\left(E\left(\sigma^{[m]}\right)-E(\sigma)\right)\right] \quad \text { (otherwise) }
\end{array} m=1, \ldots, N\right.
$$

Usually, the sequence in eq. (22) is directly simulated by means of a rejection method. The move $\sigma \rightarrow \sigma^{[m]}$ is accepted with probability eq. (3), otherwise the system stays in configuration $\sigma$. The method we use calculates the trapping times $\tau_{i}$ not by repeatedly rejecting moves, but by sampling $\tau_{i}$ from its probability distribution $P_{\sigma}(\tau)$; it then cashes in repeatedly on the initial expenditure of calculating $P_{\sigma}(\tau)$.

When one reaches a configuration $\sigma$, the transition probabilities $p_{m}=p\left(\sigma \rightarrow \sigma^{[m]}\right)$ towards all the neighboring configurations $\sigma^{[m]}, m=1, \ldots, N$ are calculated. The escape probability from configuration $\sigma$ is $p_{e s c}=\sum_{m} p_{m}$, and the probability of leaving $\sigma$ after exactly $\tau$ MC is equal to $P_{\sigma}(\tau)=p_{e s c}\left(1-p_{e s c}\right)^{(\tau-1)}$. The conditional probability for reaching configuration $\sigma^{[m]}$, given that one leaves $\sigma$, is $q_{m}=p_{m} / p_{\text {esc }}$. From $\sigma$, the algorithm samples the time $\tau$ at which the next visited configuration will be reached, using the distribution $P_{\sigma}(\tau)$. It samples independantly the new configuration $\sigma^{[m]}$ (automatically different from $\sigma$ ) by choosing the value of $m$ with probability $q_{m}$. This algorithm is exactly equivalent to the standard one.

The price one pays in this algorithm is to compute for each visited configuration all the $p_{m}, m=1, \ldots, N$, while only one is computed in the usual method. Therefore this algorithm becomes more efficient roughly speaking when the acceptance rate of the usual rejection method becomes of order $1 / \mathrm{N}$ [24]. Furthermore we have implemented in the algorithm an important feature which provides a large gain in computer time, at least on the LABS problem. We constantly keep in memory an "archive" of the different configurations visited recently during the simulation and of the corresponding probabilities $p_{m}$ for $m=1, \ldots, N$. 
Upon encountering a configuration $\sigma$ which belongs to the archive we use the $p_{m}$ in memory. In our implementation the archive is emptied automatically as soon as the number of archived configurations reaches a number $N_{\text {arch }}$. In the actual calculations described below, for the runs at the smallest temperature $T=.075$, a configuration which enters the archive (with $N_{\text {arch }} \sim 400$ ) typically remains active for a very long time (during $\sim 10^{6}$ configurations), before it is moved out.

Let us mention that the physical limit corresponding to a vanishing elementary time step $\Delta \tau \rightarrow 0$ is trivially taken in this algorithm. In this limit, the distribution of the escape time $\tau$ from the configuration $\sigma$ is

$$
P_{\sigma}(\tau)=\lambda \exp [-\lambda \tau],
$$

where $\lambda$ is related to the transition probabilities of (3) by $\lambda=\sum_{m} p\left(\sigma \rightarrow \sigma^{[m]}\right) / \Delta \tau$.

In the above method, the sampling of the dynamical evolution eq. (2) is split into two processes, the generation of the trajectories, and the sampling of physical escape times with the probability distribution $P_{\sigma}(\tau)$. These two processes are independent sources of statistical noise and the second one can be removed. Consider a trajectory $\sigma_{1}\left(\lambda_{1}\right) \rightarrow \sigma_{2}\left(\lambda_{2}\right) \ldots \rightarrow$ $\sigma_{M}\left(\lambda_{M}\right)$, where $\lambda_{i}$ is the mean escape time of configuration $\sigma_{i}$, characterizing $P_{\sigma_{i}}(\tau)$ as in eq. (4). For any realization, the physical times have to be sampled from eq. (4)). The probability $P_{M}(\tau)$ to be at configuration $\sigma_{M}$ at a given time $\tau$ is a convolution which can be computed by the calculus of residues. In the special case in which all the $\lambda_{i}$ 's are different from each other, the result takes the simple form:

$$
P_{M}(\tau)=\sum_{j=1}^{M} \sum_{i=1 \neq j}^{M} \frac{\lambda_{i}}{\lambda_{i}-\lambda_{j}}\left[1-\exp \left(-\lambda_{j} \tau\right)\right]
$$

Unfortunately, we have not succeeded in calculating this quantity in a stable way for large $M$ (such as $M \sim 10^{6} \sim 10^{8}$ ), which is the case of practical interest here. A practical answer, and the one which we have adopted, is simply to calculate, for a given trajectory eq. (2), a fair number of realizations of the escape times (with a completely unsophisticated program, we can calculate an average over $\sim 100$ realizations of the escape times in just about twice the time it takes to generate the trajectory).

We have used this algorithm to study the LABS model. We begin with the results from simulated annealing. In fig. 1 we plot the internal energy per spin vs temperature at $N=100$. The annealing scheme starts from a temperature $T_{1}=.6$. After thermalizing at this temperature, the temperature is lowered down to $T_{0}=.04$ using the logarithmic scheme: $T(t)=T_{1}-\left(T_{1}-T_{0}\right) \log (t) / \log \left(t_{m}\right)$, where the duration of the run, $t_{m}$ (measured in Monte Carlo steps per Spin (MCS)), takes the values $1.25^{20}, 1.5^{20}, 1.75^{20}, 2^{20}, 2.25^{20}, 2.5^{20}$. So the logarithmic cooling rate $d T / d(\ln (t))$ varies from .125 to .0306 . The data is averaged over 400 to 50 realizations of the random process of eq. (2) with random initial conditions; for each sequence of configurations we have studied a single realization of the escape times. The system undergoes a freezing phenomenon at a temperature which depends on the logarithmic cooling rate, reminiscent of the observations in glasses. An extrapolation of the zero-temperature energy as a function of the logarithmic cooling rate is compatible with a zero-temperature energy per spin of the order of $.07 \sim .08$. This result is about twice as large as the value $E_{c}=.0406 . E_{c}$ is conjectured by the Bernasconi-Golay approximation [5. 18], 
recently derived using an approximate description of this system by one with quenched disorder [20], and is also compatible with exact enumerations of small size systems. We have done simulations at $N=200, N=400$ and $N=401$, and $t_{m}=1.25^{20}, 1.5^{20}, 1.75^{20}, 2^{20}$ which are nearly indistinguishable from the data of fig. 1 . The difference between the result of simulated annealing and $E_{c}$ may well be due to a dynamic freezing effect, which forbids to find the exact ground state for large size systems.

We have performed a more detailed study of the dynamics at temperatures $T=.075$, which is clearly in the low $T$ regime on all the time scales we can reach, and at $T=.25$ for comparison. We start by reviewing the results at the lower temperature, $T=.075$. In fig. 2 we plot the correlation function $C\left(t_{w}+\tau, t_{w}\right)=(1 / N) \sum_{i}<\sigma_{i}\left(t_{w}+\tau\right) \sigma_{i}\left(t_{w}\right)>$ $v s$ the time $\tau$ for various waiting times $t_{w}$, ranging up to $10^{7}$ (from now on all the times are measured in MCS). The aging effect is clear, on all the range of waiting times we can reach. The behavior is well approximated by a function of $\tau / t_{w}$, decreasing as a power law $\left(\tau / t_{w}\right)^{-.026}$ at large arguments, as shown in fig. 3. This is similar to what has been found in experiments [2], and numerical simulations of spin glasses [4] or other disordered systems [25]. Recent analytical studies of some mean field spin glasses confirm the possibility of such a scaling [11] for a category of spin glasses characterized by a first order ("one step") replica symmetry breaking scheme like the Random Energy Model [22.223], while the spin glasses with full replica symmetry breaking seem to have a more complicated behavior [12, 13]. We exhibit here a clear aging effect for the LABS model which is a system without any quenched disorder. This data again suggests that the LABS could be a good toy model for the glass transition.

On this aging data one also notices that the asymptotic dynamics is essentially frozen: $\lim _{\tau \rightarrow \infty} \lim _{t_{w} \rightarrow \infty} C\left(t_{w}+\tau, t_{w}\right) \simeq 1$. It is interesting to look at a single run of a system with $N=400$ spins, at $T=0.075$. We show such a run, typical of what we observe, in fig. 4. The simulation, which has taken a few hours on a HP 730 workstation, was stopped after a time of $\tau=10^{9}$ MCS. It is immediately seen on the plot of the energy vs time, that the energy of the system is not equilibrating on this time scale. Clearly the system gets trapped into metastable states with a long lifetime which are well identified by the plateaux in the energy. The fluctuations along each plateau are quite small, which is in agreement with the above observations: the metastable states at low temperature involve a small number of configurations. In contrast we show in figs 5 and 6 the analogous data at a higher temperature $T=.25$. There is no trace of the plateaux in the energy, and the system equilibrates after a finite time $t \simeq 10^{3}$, which agrees with the simulated anealing data from fig. 1. On the correlation function one observes an interrupted aging effect.

The trapping behavior is reminiscent of the simple generic model of aging proposed by Bouchaud [8]. At a qualitative level one does see on fig. 4 that the trapping times (length of the plateaux) become longer when the waiting time increases. A quantity which is easily accessible with our algorithm is the distribution of escape time from single configurations $\tilde{\tau}=1 / \lambda$. (Notice that this is not exactly the same as the trapping times because the plateaux involve several configurations). In fig. 7 we plot $\int_{\tau}^{\infty} P_{k}\left(\tau^{\prime}\right) d \tau^{\prime} v s \tau$, where $P_{k}\left(\tau^{\prime}\right)$ is the distribution of escape time for the $k$ th visited configuration in the sequence eq. (2)). We see a clear increase of the typical escape time with $k$ (and therefore with the age of the system). For large waiting time (large $k$ ) the distribution $P_{k}\left(\tau^{\prime}\right)$ is broad, and compatible with the idea of [8] that the average escape time is infinite. The behavior of $P_{k}(\tau)$ at large times 
$\tau$ can be approximated by a power law $P_{k}(\tau) \simeq \tau^{-1.6}$. The broadness of this distribution explains why the algorithm we use is so efficient on this problem. It circumvents the problem of trapping by a single configuration, but does not get rid of "renormalized traps" consisting of a group of configurations separated from the rest by barriers. A cluster Monte Carlo algorithm [26] has been developed to handle this problem.

To conclude, we have found a model without quenched disorder which is another representative of a well known class of glassy systems, those which undergo a "one step replica symmetry breaking" transition in the static approach. Other members of this class include the Random Energy Model [22,23], the binary perceptron [27], the p-spin spherical spin glass [28], random heteropolymers [29 31], the manifolds in random media with short range disorder [32], etc. We believe that the algorithm we have described will be efficient for any of these problems. Our code is available by e-mail.

\section{ACKNOWLEDGMENTS}

We acknowledge helpful discussions with J.P. Bouchaud, E. Marinari and G. Parisi.

[1] A. B. Bortz, M. H. Kalos, J. L. Lebowitz J. Comput. Phys. 17, 10 (1975) cf also : K. Binder in Monte Carlo Methods in Statistical Physics, edited by K. Binder, 2nd ed. (Springer Verlag, Berlin, 1986) sect 1.3.1

[2] L. Lundgren, P. Svedlindh, P. Nordblad and O. Beckman, Phys. Rev. Lett. 51 (1983) 911;

R. V. Chamberlin, G. Mozurkevich and R. Orbach, Phys. Rev. Lett. 52 (1984) 867; M. Alba, J. Hamman, M. Ocio and Ph. Refrigier, J. Appl. Phys. 61 (1987) 3683;

[3] M. Lederman, R. Orbach, J. M. Hamman, M. Ocio and E. Vincent Phys. Rev. B44 (1991) 7403; J. M. Hamman, M. Lederman, M. Ocio, R. Orbach and E. Vincent Physica A 185 (1992) 278;

[4] J. O. Anderson, J. Mattson, and P. Svedlindh, Phys. Rev. B46 (1992) 8297; H. Rieger, J. Phys. A 26 (1993) L615; L. Cugliandolo, J. Kurchan and F. Ritort, Phys. Rev. B49 (1994) 6331 ;

[5] J. Bernasconi, J. Physique 48559 (1987);

[6] G. J. M. Koper and H. J. Hilhorst, J. Phys. France 49 (1988) 429;

[7] D. S. Fisher and D.A. Huse, Phys. Rev. B38 (1988) 373;

[8] J. P. Bouchaud, J. Phys. I 2 (1992) 1705; J. P. Bouchaud, E. Vincent and J. Hamman, J. Phys. France 4 (1994) 139;

[9] H. Horner, Z. Phys. B66 (1987) 175;

[10] L. B. Ioffe, Phys. Rev. B38 (1988) 5181 ; V. S. Dotsenko, M. V. Feigelman and L. B. Ioffe, Soviet scientific reviews 15 (1990) 1;

[11] L.F. Cugliandolo and J. Kurchan,Phys. Rev. Lett. 71 (1993) 173;

[12] S. Franz and M. Mzard, Europhys. Lett. 26 (1994) 209; Physica A209 (1994) 1; 
[13] L.F. Cugliandolo and J. Kurchan, On the out of equilibrium relaxation of the Sherrington Kirkpatrick model, Rome University preprint 977, cond-mat 9311016;

[14] W. Gotze, in 'Liquids, freezing and glass transition', Les Houches 1989, J. P.Hansen, D. Levesque, J. Zinn-Justin Editors, North Holland1991;

[15] P. Goldbart and A. Zippelius, preprint (1994);

[16] J. D. Shore, M. Holzer, J. P. Sethna; Phys Rev. B 4611376 (1992)

[17] H. Eissfeller and W. Kinzel, J. Phys A Math Gen 251473 (1992)

[18] M. J. E. Golay, IEEE IT-23 (1977) 43, IEEE IT-28 (1982) 543;

[19] G. Migliorini, tesi di laurea, Università di Roma II, 1993 (unpublished), and in preparation.

[20] J. P. Bouchaud and M. Mézard, preprint LPTENS 94/14, to appear in J. Phys. France, August 1994 .

[21] E. Marinari, G. Parisi and F. Ritort, preprints Università di Roma I, may 1994;

[22] B. Derrida, Phys. Rev. B 24 (1981) 2613;

[23] D. Gross and M. Mézard, Nucl. Phys. B 240 (1984) 431

[24] Let us stress that the true problem, of course, is not a uniformly low acceptance probability (which could be repaired by simply choosing a large value of $\Delta \tau$ ), but the existence of dynamic relaxations on a variety of time scales.

[25] E. Marinari and G. Parisi, J. Phys. A 26 (1993) L1149

[26] W. Krauth and O. Pluchery, A Rapid Dynamical Monte Carlo Algorithm for Glassy Systems, LPS preprint 1994

[27] W. Krauth and M. Mézard, J. Phys. France 503057 (1989)

[28] T. R. Kirkpatrick and D. Thirumalai, Phys. Rev. Lett. 58 (1987) 2091, Phys. Rev. B38 (1987) 5388 ;

[29] T. Garel, H. Orland, Europhys. Lett.6, 307 (1988);

[30] E. I. Shakhnovich and A. M. Gutin, Europhys. Lett. 8, 327 (1989);

[31] G. Iori, E. Marinari and G. Parisi, J. Phys. A24 (1991) 5349;

[32] M. Mézard and G. Parisi, J. Physique I (1991)809.

\section{FIGURE CAPTIONS}

Fig. 1: Simulated annealing results of the energy per spin vs temperature in a $N=$ 100 spin system, corresponding to logarithmic cooling rates (from top to bottom) $.125, .07, .05, .04, .034, .03$.

Fig. 2: The two times correlation of a $N=400$ spin system at temperature $T=.075$, $C\left(t_{w}+\tau, t_{w}\right)$ vs $\tau$, plotted for various values of the waiting time $t_{w}=5^{n}$ with $n=$ $0,1, \ldots, 10$. The data is averaged over 300 initial conditions (and for each of them 50 realizations of the sampling of escape times).

Fig. 3: The same data as fig.2 plotted $v s \tau / t_{w}$, for $t_{w}=5^{2 n}$ with $n=0,1, \ldots, 5$.

Fig. 4: Energy per spin vs $\log _{10}$ (time) in a single run for a $N=400$ spin system at $T=.075$.

Fig. 5: The two times correlation of a $N=400$ spin system at temperature $T=.25$, $C\left(t_{w}+\tau, t_{w}\right)$ vs $\tau$, plotted for various values of the waiting time $t_{w}=2.5^{n}$ with 
$n=0,1, \ldots, 7$. The data is averaged over 400 initial conditions (and for each of them 50 realizations of the sampling of escape times).

Fig. 6: Energy per spin vs $\log _{10}$ (time) in a single run for a $N=400$ spin system at $T=.25$.

Fig. 7: The integrated distribution of escape times, $\int_{\tau}^{\infty} P_{k}\left(\tau^{\prime}\right) d \tau^{\prime}$, for the $k$ th visited configuration, is plotted vs $\tau$ for $k=4^{n}$ with $n=2, \ldots, 7$, in a simulation of a $N=400$ spin system at $T=.075$. 


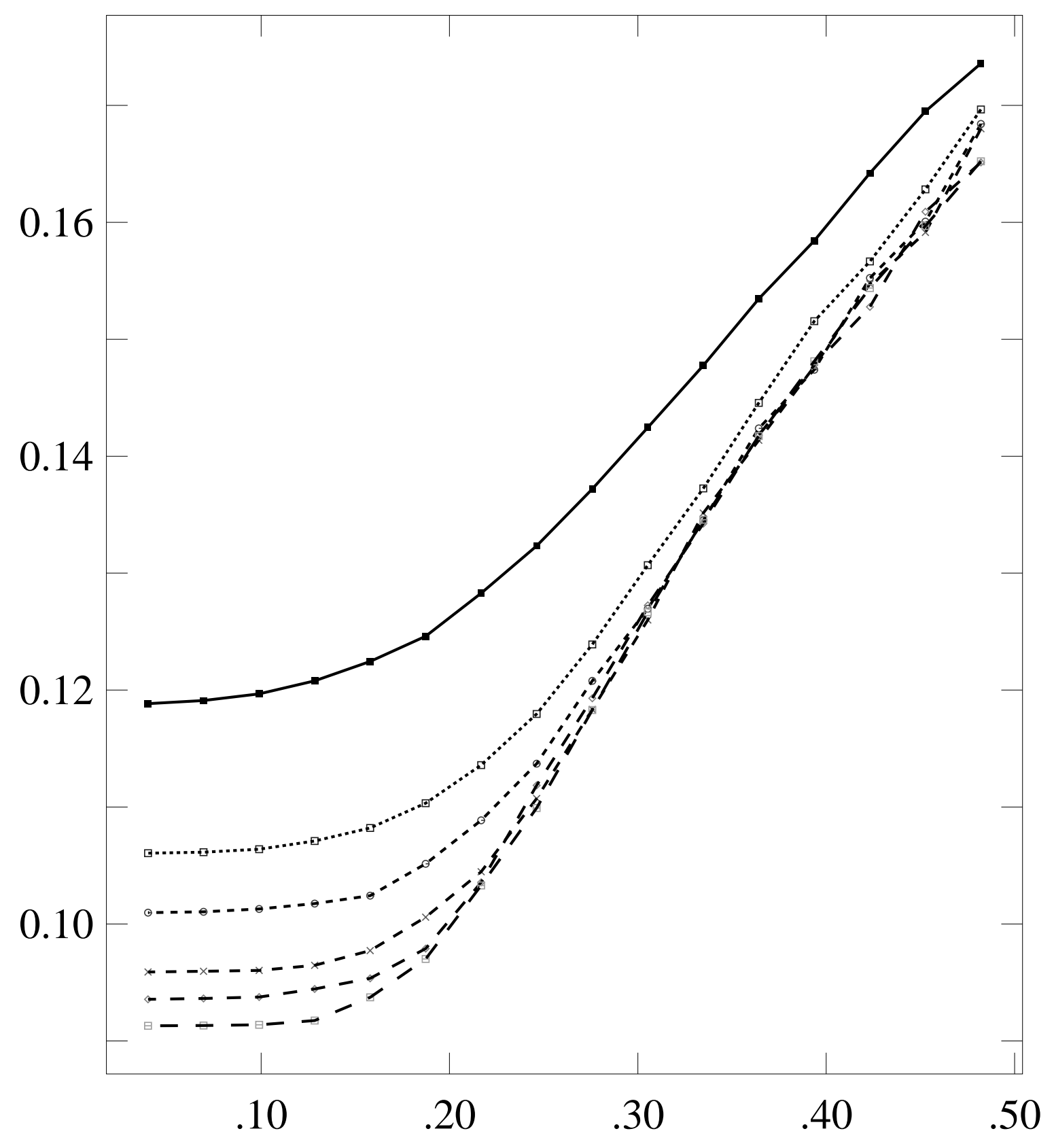




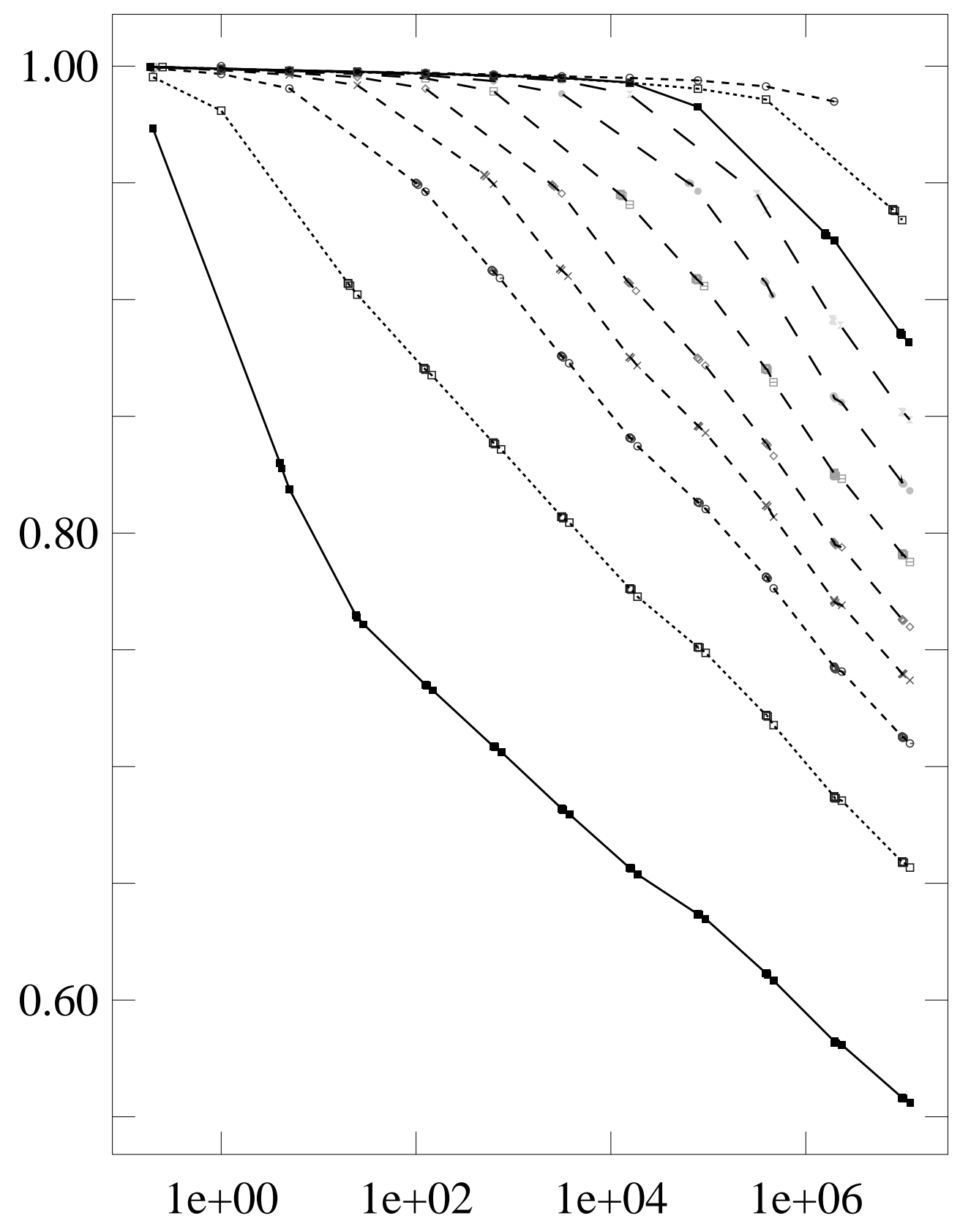




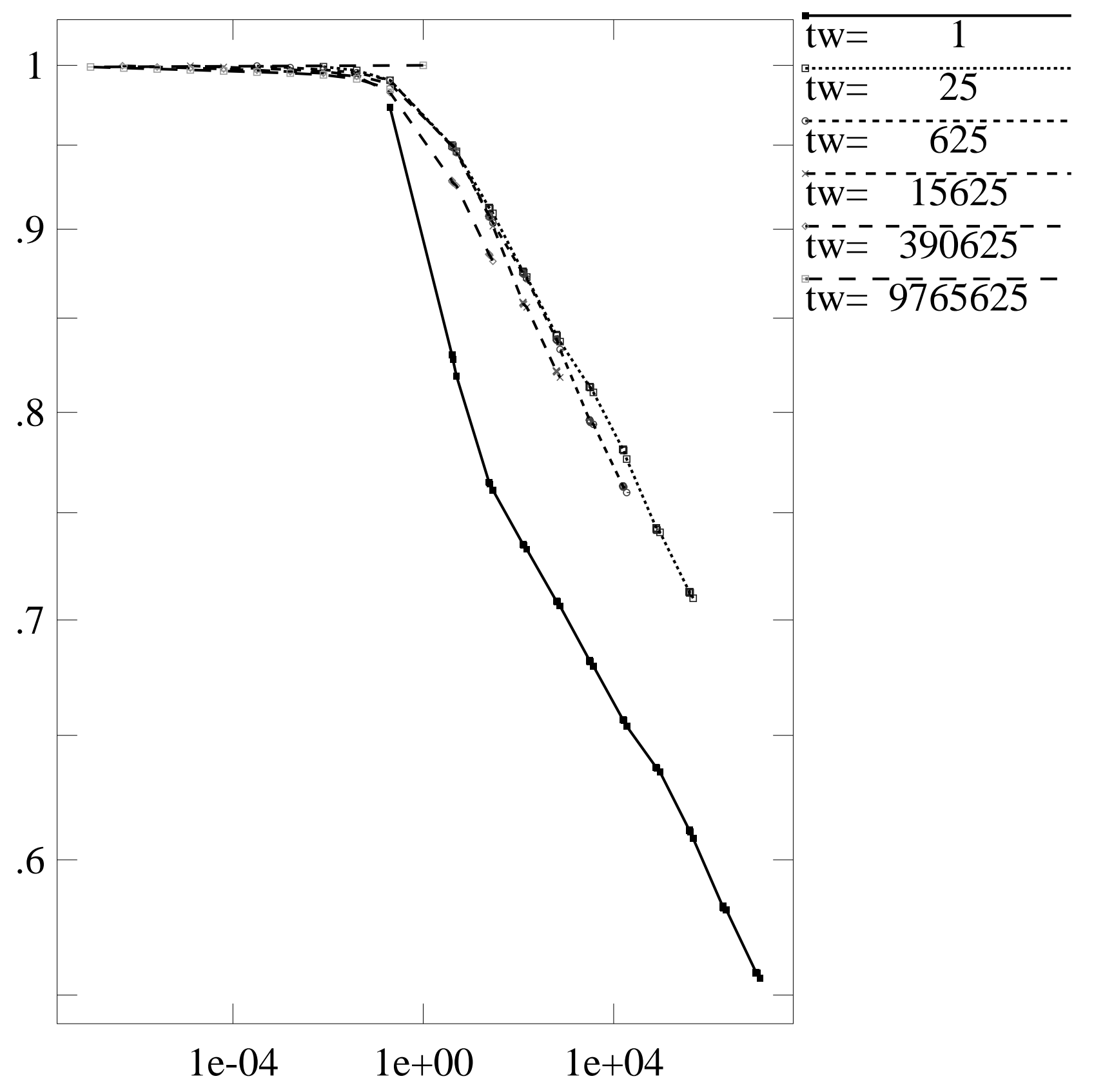




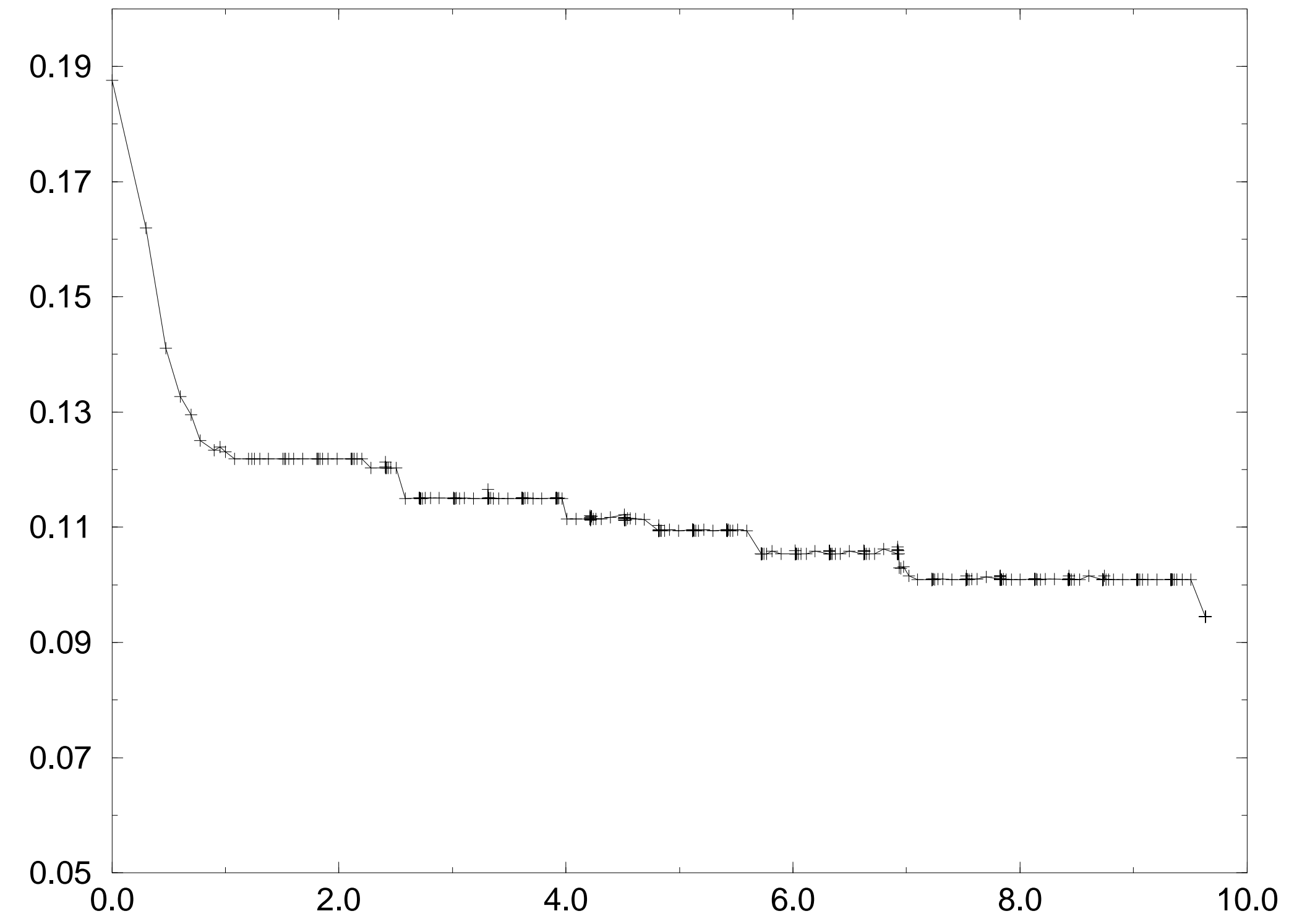




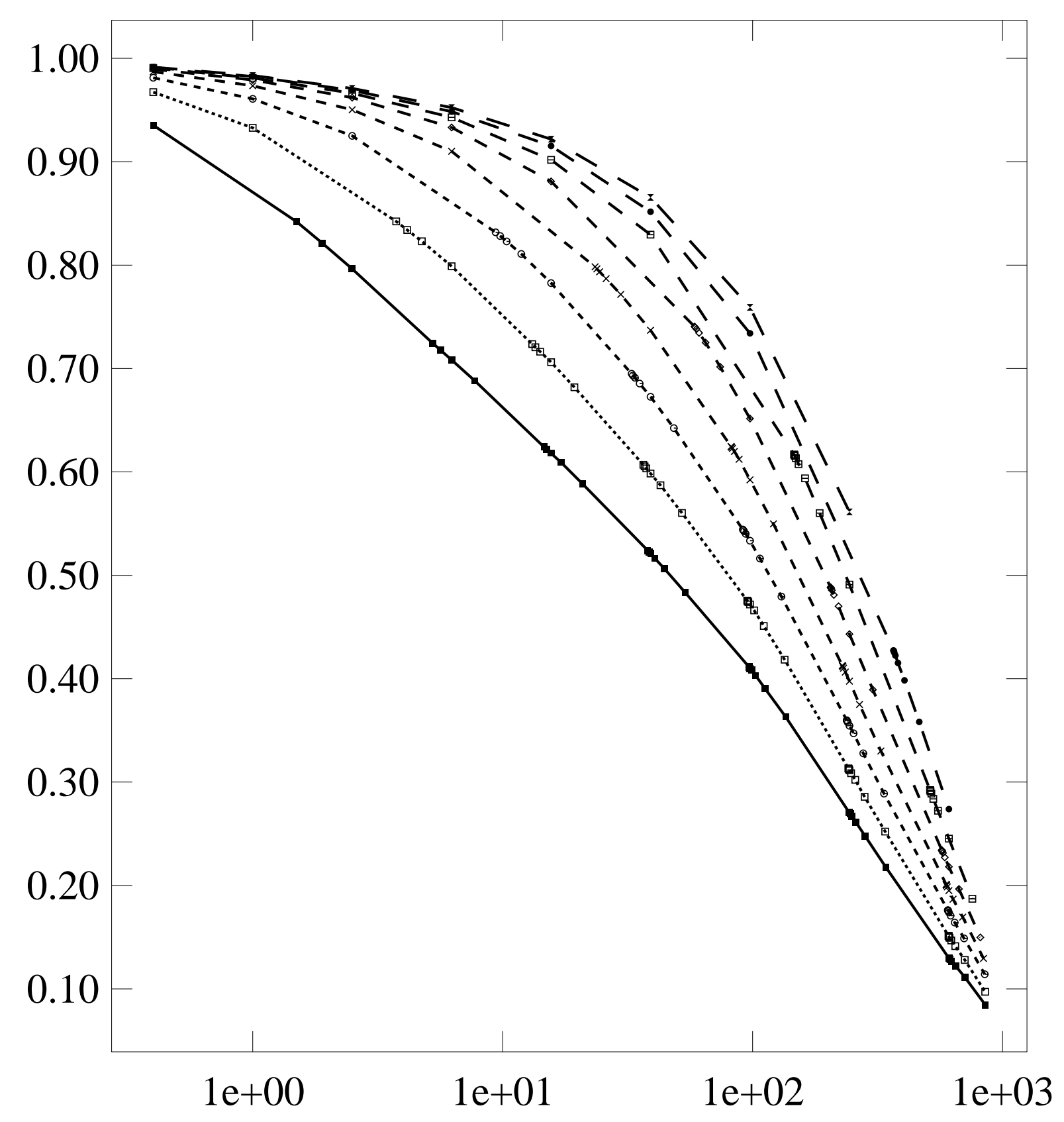




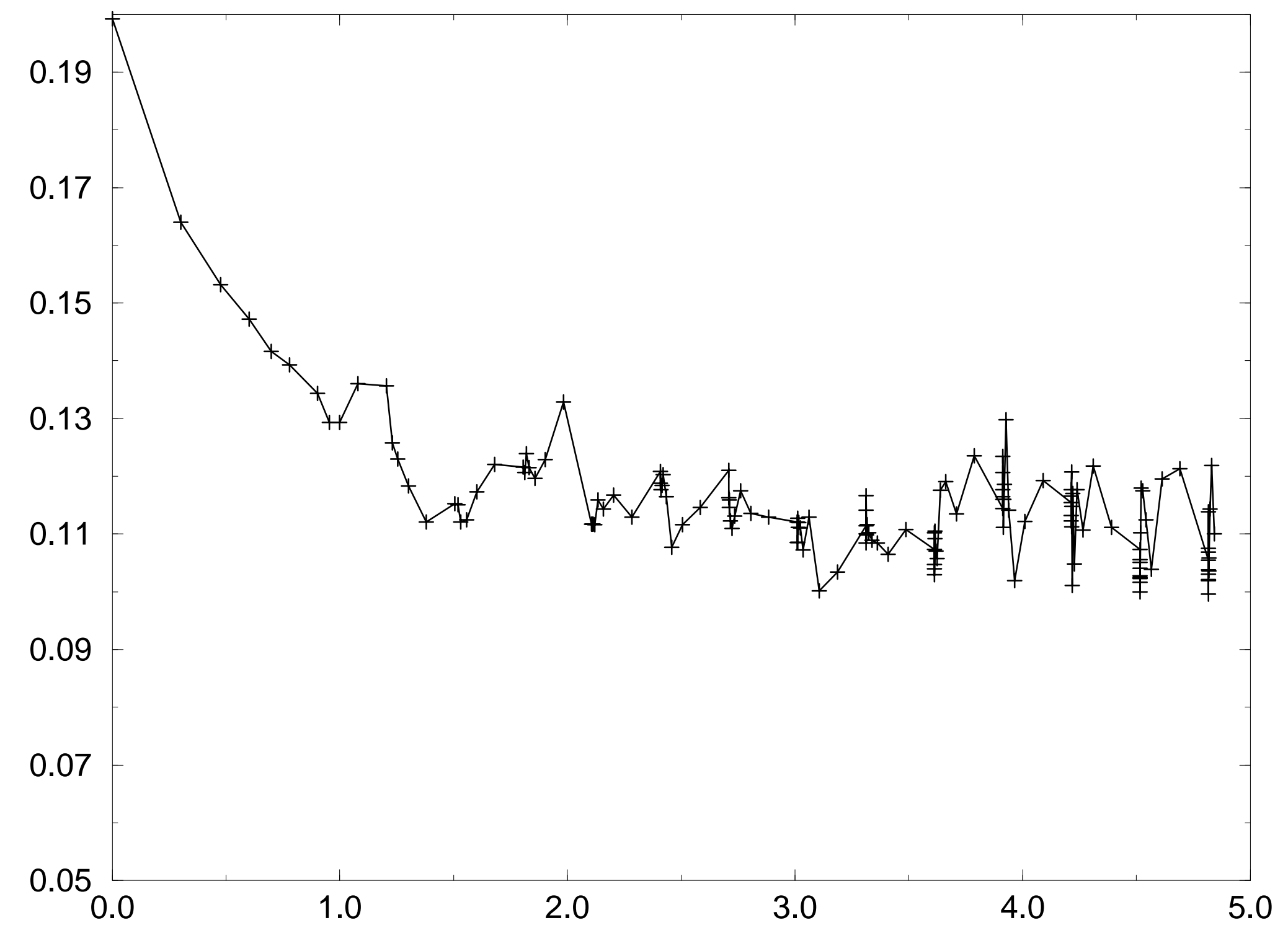




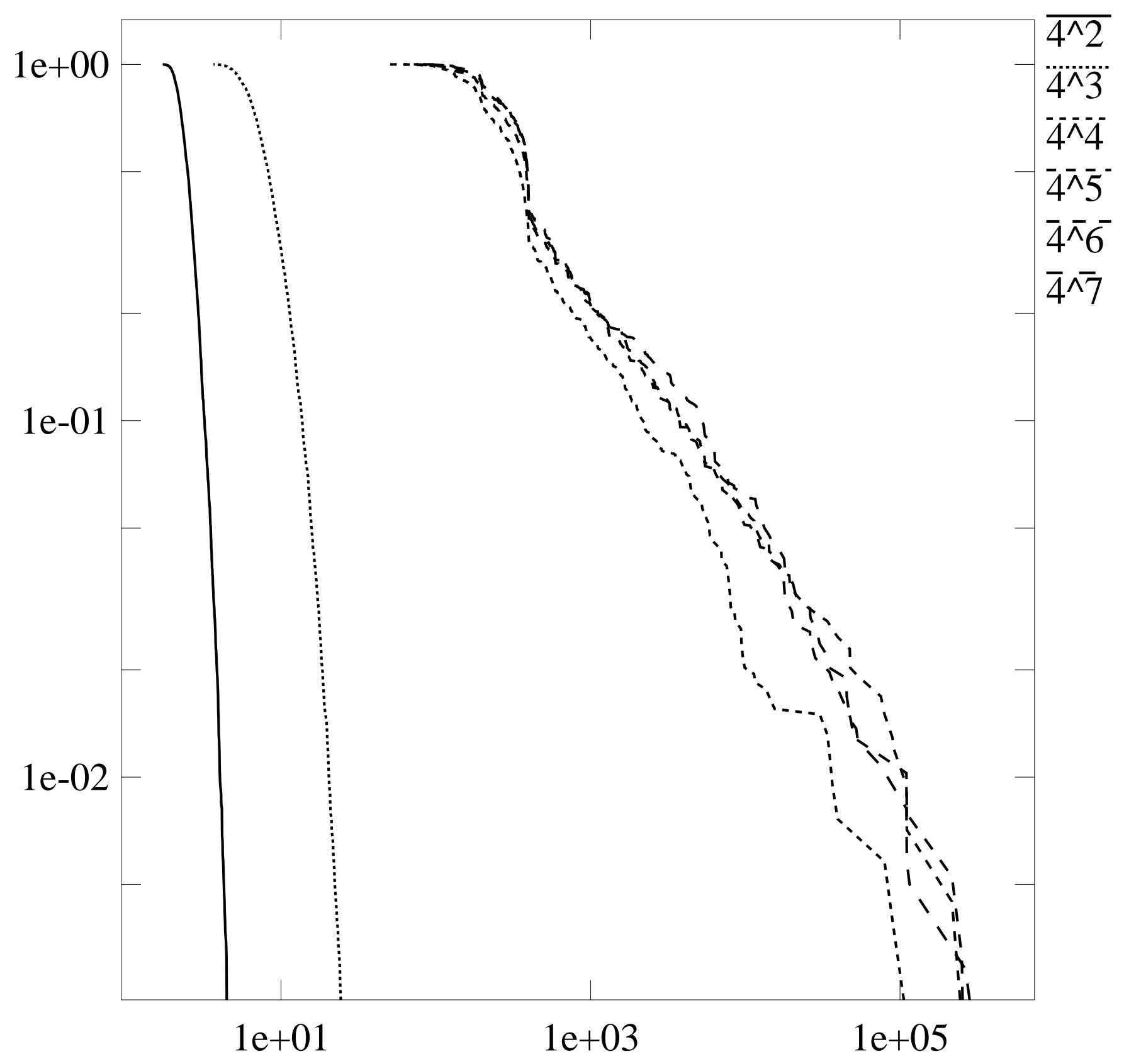

